\title{
Thermodynamics of pseudospin-electron model in mean field approximation
}

\author{
I.V.Stasyuk, O.D.Danyliv \\ Institute for Condensed Matter Physics \\ 1 Svientsitskii str., Lviv 290011, Ukraine \\ tel./fax: + 380 (322) 761978 \\ $6 / 10 / 1999$
}

PACS 05.70.-a; 74.72.Bk; 71.10.Fd

Keywords: Hubbard model, local anharmonicity, phase transitions

\begin{abstract}
The mean field type approach based on the self-consistent consideration of an effective field created by electron transfer is developed for a description of thermodynamics of the Hubbard type models with an infinitely large on-site repulsion. This procedure, formulated according to [1], is an extension of the recently proposed generalized random phase approximation(GRPA).

Within this approach, the thermodynamic properties of the two-sublattice pseudospin-electron model (the Hubbard model with local anharmonicity) are studied. Such a model can be used for a description of dielectric properties of YBaCuO-type superconductors along $c$-axis; pseudospins represent anharmonic motions of apical oxygens O4. It is shown that there are either phase transitions in the model with jumps of the mean values of a pseudospin and of electron concentration (in the $\mu=$ const regime) or the phase separation (in the $n=$ const regime). The phase transitions or phase separation are caused by pseudospin-pseudospin interaction as well as by electron transfer (the latter results in appearing of effective interaction between pseudospins). The possibility of the ferroelectric type ordering of pseudospins is investigated.
\end{abstract}

\section{Introduction}

The Hubbard model [2] is a fundamental one in the theory of strongly correlated electron systems. After the discovery of high-temperature superconductors it has been intensively studied, because the HTSC-compounds necessarily possess planes with strong correlation between electrons described by this model. Amongst the most laborious but promising approaches to study the Hubbard model there is a diagrammatic expansion with respect to atomic limit, which essentially differs from the diagrammatic technique for Fermisystems [3]. One of recently worked out developments is the GRPA proposed by Izyumov 
et.al. in order to study the Hubbard model with Coulomb repulsion $U=\infty$ [4]- 6 . Application of the GRPA approach to a pseudospin-electron system can be found in [7][9]. However, this approach, being developed for investigation of correlation functions, is not a self-consistent theory. Therefore, lattice instabilities within it are treated as a result of singularities of susceptibilities that cannot suffice when the model undergoes the first order phase transition. The question how to calculate the thermodynamic quantities within the GRPA remains open as well.

Returning to the possible approaches to a description of models with strong electron correlation, one should note that due to a success in obtaining exact results for simple models (Falicov-Kimball model [10], [11], pseudospin-electron model with $U=0$ [12], etc), the approximation of the infinite dimension of space becomes very popular. However, there are some problems here, too. The single-site problem for the Hubbard model remains analytically unsolved; there are some difficulties with obtaining the higher order expansions in powers of $1 / d$.

In this paper we use the mean field approximation, proposed in [1] for investigation of the $t-J$ model. We show using as an example the Hubbard model that the diagrammatic series, summed over in this approximation expands the diagrammatic series of the GRPA, and, hence, we can talk about the self-consistent approach within the GRPA. The approximation is applied to the two-sublattice pseudospin-electron model which, being derived for description of ferroelectric type effects in HTSC of $\mathrm{YBaCuO}$ type (see [13][17]), takes into account the two-sublattice structure of an apex oxygen subsystem in such superconductors. Coupling between the electron and pseudospin subsystems ( pseudospin variables are used to describe the local strongly anharmonic vibrations of apex oxygens) is performed via an interaction of the $g n_{i} S_{i}^{z}$ type proposed by Müller [18].

The paper is composed as follows. In Section 2 the method of construction of mean field approximation within the GRPA scheme for the Hubbard model with $U=\infty$ is presented. Application of the method to the two-sublattice pseudospin-electron model is given in Section 3. Sections 4 and 5 contain the results of numeric calculations and conclusions, respectively.

\section{MFA approximation applied for Hubbard model with infinite Coulomb repulsion}

In this section we show how to obtain the MFA equations in example case of one-band Hubbard model and discuss the accuracy of that approach. The Hamiltonian of the Hubbard model without taking into account the influence of magnetic field reads

$$
H=-\mu \sum_{i, s} n_{i}^{s}+U \sum_{i} n_{i}^{\uparrow} n_{i}^{\downarrow}+\sum_{i, j, s} t_{i j} a_{i s}^{+} a_{j s}
$$

where $\mu$ is the chemical potential; $U$ is a Coulomb repulsion of electrons on the same orbital; $t_{i j}$ describes an electron transfer from the site $j$ to the site $i$ of a real lattice. $a_{i s}$ is the annihilation operator of an electron with spin $s(s$ takes two values $-\uparrow$ and $\downarrow$ ), $n_{i}^{s}=a_{i s}^{+} a_{i s}$ is the electron number operator. 
Usually near the atomic limit the model is considered using the basis of four states $\left|R_{i}\right\rangle \equiv\left|n_{i}^{\uparrow}, n_{i}^{\downarrow}\right\rangle$

$$
\begin{aligned}
& |1\rangle=|0,0\rangle \\
& |2\rangle=|1,1\rangle \\
& |3\rangle=|0,1\rangle \\
& |4\rangle=|1,0\rangle
\end{aligned}
$$

and Hubbard operators which are defined as follows $X_{i}^{R S}=\left|R_{i}\right\rangle\left\langle S_{i}\right|$.

Let us introduce the "bare" (zero-order) Matsubara Green's function as [19]

$$
\begin{aligned}
& g_{i j}^{p q}\left(\tau-\tau^{\prime}\right)=\delta_{i j} \frac{\left\langle T X_{i}^{p q}(\tau) X_{j}^{q p}\left(\tau^{\prime}\right)\right\rangle_{0}}{\left\langle\left[X_{i}^{p q}, X_{j}^{q p}\right]_{ \pm}\right\rangle_{0}}=\delta_{i j} \mathrm{e}^{\varepsilon^{p q}\left(\tau-\tau^{\prime}\right)} \\
& n_{ \pm}\left(\varepsilon^{p q}\right)=\frac{1}{\mathrm{e}^{\beta \varepsilon^{p q}} \pm 1}, \quad \varepsilon^{p q}=\varepsilon^{p}-\varepsilon^{q}
\end{aligned}\left\{\begin{array}{l} 
\pm n_{ \pm}\left(\varepsilon^{p q}\right), \quad \tau>\tau^{\prime} \\
\pm n_{ \pm}\left(\varepsilon^{p q}\right)-1, \quad \tau<\tau^{\prime}
\end{array}\right.
$$

where $T$ means $T$-product, $X_{i}^{p q}(\tau)=\mathrm{e}^{\tau H_{0}} X_{i}^{p q} \mathrm{e}^{-\tau H_{0}}$ is an operator in interaction representation and $\langle\ldots\rangle_{0}$ is the average with the Gibbs distribution with the zero-order Hamiltonian. $\varepsilon^{p}$ are the single-site energy levels of the zero-order Hamiltonian (at $\left.t_{i j}=0\right)$ :

$$
\varepsilon^{1}=0, \quad \varepsilon^{2}=-2 \mu+U, \varepsilon^{3}=\varepsilon^{4}=-\mu .
$$

The upper sign is chosen when the operators $X_{i}^{p q}$ are of the Fermi-type $\left(X^{41}, X^{23}, X^{31}\right.$, $X^{24}$ and complex conjugate); otherwise, the lower sign should be taken. Correspondingly, $[\ldots]_{+}$is an anticommutator, and $[\ldots]_{-}$is a commutator.

The results of perturbation theory and applying of Wick's thorem [19, 20] can be presented by elements which are denoted in the following way

$$
\begin{aligned}
& g_{i j}^{p q}\left(\tau-\tau^{\prime}\right)=\stackrel{i}{\mathrm{pq} \gamma^{j}} \\
& \left\langle X_{i}^{p p}\right\rangle_{0}=\text { P }, \quad\left\langle X_{i}^{p p} X_{i}^{q q}\right\rangle_{0}^{c} \equiv \frac{\partial}{\partial\left(-\beta \varepsilon^{p}\right)}\left\langle X_{i}^{q q}\right\rangle_{0}=\text { (1) (9) } \\
& \left\langle X_{i}^{p p} X_{i}^{q q} X_{i}^{m m}\right\rangle_{0}^{c} \equiv \frac{\partial}{\partial\left(-\beta \varepsilon^{p}\right)} \frac{\partial}{\partial\left(-\beta \varepsilon^{q}\right)}\left\langle X_{i}^{m m}\right\rangle_{0}=(\overline{\mathrm{D}} \text { (9) } \\
& g_{i j}^{p q}\left(\tau-\tau^{\prime}\right)\left\langle X_{i}^{p p}+X_{i}^{q q}\right\rangle \equiv g_{i j}^{p q}\left(\tau-\tau^{\prime}\right) B_{i}^{p q}=\stackrel{i}{\mathrm{pq}} \bigcirc^{j} \\
& t_{i j}={ }^{i} \sim \sim^{j}
\end{aligned}
$$

Let us write here the diagrammatic expansion for the average of $X$-operator in Heisenberg representation $\left\langle T \tilde{X}_{i}^{11}(\tau)\right\rangle=\left\langle T \mathrm{e}^{\tau H} X_{i}^{11} \mathrm{e}^{-\tau H}\right\rangle, i=1$ : 

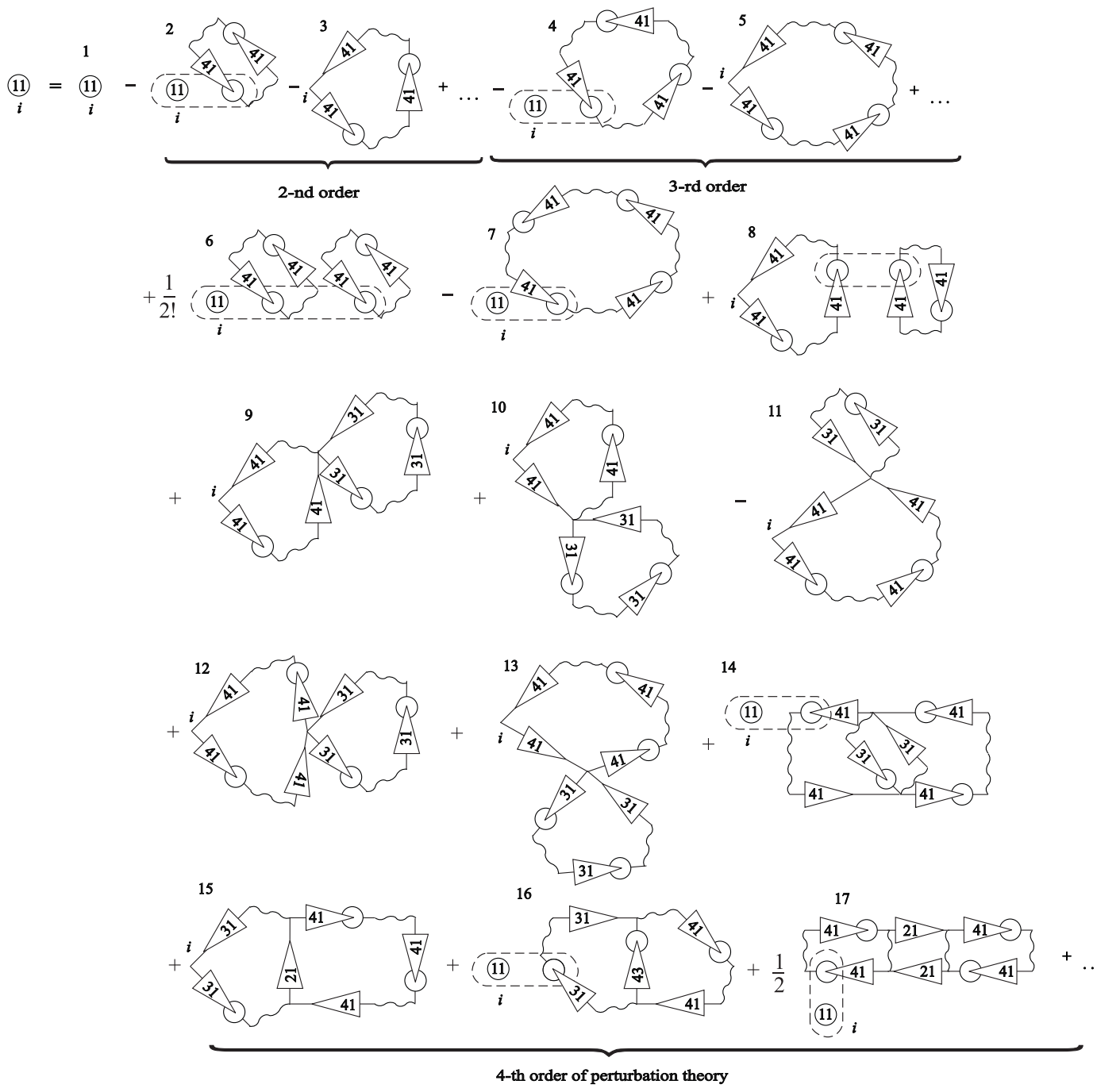

Retaining the diagrams with only simple loops, we can neglect the diagrams which contain the Green's functions $g_{i j}^{43}\left(\tau-\tau^{\prime}\right)$. In the limiting case $U=\infty$, which we shall consider hereafter, we can neglect the Fermi-type Green's functions $g_{i j}^{24}, g_{i j}^{23}$ and the Bosetype Green's function $g_{i j}^{21}$ as well. Discarding the mentioned class of diagrams, one can show that the results of the application of Wick's theorem do not depend on the so-called priority rules [5].

Classifying the obtained result, we treat the diagrams 2, 4, 6, 7 as a "thickening" of the zero-order average $\left\langle X^{11}\right\rangle_{0}$ by the insets of the mean field type. The three last diagrams in : should be discarded from the reasons presented above. There are also other two important type of diagrams: the diagrams 9, 10 renormalize the vertex parts of the Green's function of the initial diagram 3, whereas the diagrams like 11, 12, 13 can be interpreted as an complication of self-energy parts in the more simple diagram 3 . After Fourier transformation all of the mentioned diagrams (unlike the diagrams 14-17) correspond to the one sum (or to a certain power of the one sum) over the wavevector $\mathbf{k}$ and frequency $\omega$. As one can see from the figure, the diagrams 3, 5, 9-13 are those which are summed over within the GRPA when an equation of the Bete-Salpeter type is solved [5], [9]. 
Let us introduce a set of diagrams corresponding to the generalized Hubbard-I approximation (in the simplified version that is valid in the case of independent subbands [8]), denoting it by a "thickened" Green's function.

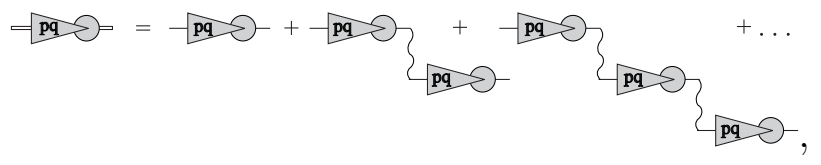

where $-p q-\equiv \tilde{g}^{p q}(\omega) \tilde{B}^{p q}$ is the "dressed" Green's functions with the renormalized vertex part. We shall consider only the uniform phase in which all sites are equivalent. Then the equation for the vertex part $\tilde{B}^{p q}$ can be written as

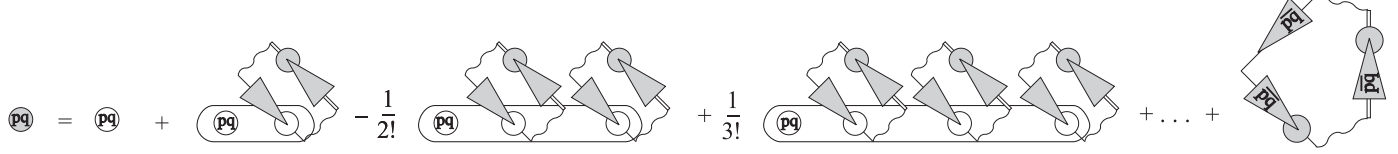

Via $\overline{p q}$ we denoted the change of a spin projection in diagrams. Thus $\overline{41}=31$, and vice versa, $\overline{31}=41$. "Dressed" Green's functions with semiinvariants $-O$ are of $\tilde{g}^{41}(\omega) \tilde{B}^{41}$ or $\tilde{g}^{31}(\omega) \tilde{B}^{31}$ type. They creates loops with spin $\uparrow$ or $\downarrow$, respectively. The series in the right hand side of the equation is analogous to the series which arises in the mean field theory for the Ising or Heisenberg model.

Formally, expression (7) can be presented as

$$
\tilde{B}^{p q}=\frac{1}{Z} S p\left(\hat{B}_{i}^{p q} \mathrm{e}^{-\beta H_{e f f}^{i}}\right)-\tilde{L}^{\overline{p q}}
$$

where

$$
\begin{aligned}
& H_{e f f}^{i}=H_{0}+\tilde{Q}^{41} \hat{B}_{i}^{41}+\tilde{Q}^{31} \hat{B}_{i}^{31} \equiv H_{0}+\sum_{\{p q\}} \tilde{Q}^{p q} \hat{B}_{i}^{p q}, \quad\{p q\}=41,31
\end{aligned}
$$

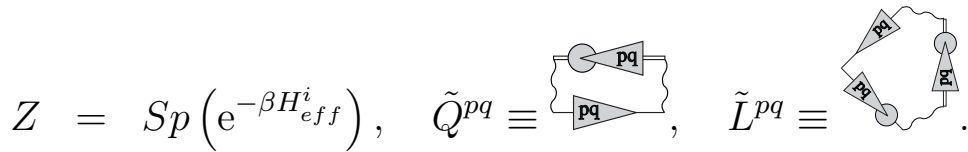

The renormalized Green's functions $\tilde{g}^{p q}(\omega)$ is defined by the equation

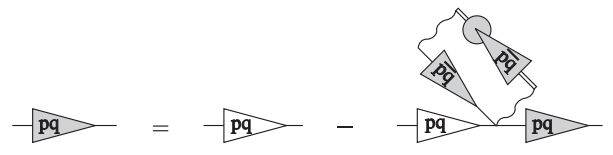

Hence

$\tilde{g}^{p q}=\frac{1}{i \omega-\varepsilon^{p q}+\tilde{Q}^{\overline{p q}}} \equiv \frac{1}{i \omega-\tilde{\varepsilon}^{p q}}, \quad \tilde{\varepsilon}^{p q}=\varepsilon^{p}-\varepsilon^{q}-\tilde{Q}^{\overline{p q}}$

That corresponds to the summation of diagrams of the 11, 12, 13 type. The expression (11) describes a renormalization of the single-electron excitation spectrum. $\tilde{Q}^{p q}$ plays the role of the irreducible self-energy part, by which the levels of the single-electron transitions are shifted. Using the renormalized Green's functions one can construct the loops $\tilde{Q}^{p q}$ 
and $\tilde{L}^{p q}$ and, thereby, derive an expression for the irreducible part and equation for the auxiliary quantity $\tilde{L}^{p q}$ :

$$
\begin{aligned}
\tilde{Q}^{p q} & =\frac{1}{N} \sum_{k} t_{k} n_{+}\left(\tilde{\varepsilon}^{p q}+t_{k} \tilde{B}^{p q}\right) \quad\left(n_{+}(\varepsilon)=\frac{1}{1+\mathrm{e}^{\beta \varepsilon}}\right) \\
\tilde{L}^{p q} & =\frac{1}{N} \sum_{k} n_{+}\left(\tilde{\varepsilon}^{p q}+t_{k} \tilde{B}^{p q}\right)-n_{+}\left(\tilde{\varepsilon}^{p q}\right) .
\end{aligned}
$$

The expression for the free energy was obtained with making use of the analogy between the diagrammatic series for $\tilde{B}^{p q}$ and series for the magnetization in the mean field theory for spin systems. Then so called one-tail diagrams correspond to simple loops in current approximation. One can show that the free energy can be written as

$$
\begin{aligned}
F & =\mu n-T \ln S p\left(\mathrm{e}^{-\beta H_{e f f}}\right)-\sum_{\{p q\}} \tilde{Q}^{p q} \tilde{B}^{p q}+\sum_{\{p q\}} \tilde{D}^{p q}, \\
\tilde{D}^{p q} & =\frac{1}{2} \sum_{p q}^{p q}+\frac{1}{3} \\
& =\frac{1}{\beta N} \sum_{k} \ln \left(1+\mathrm{e}^{-\beta \tilde{\varepsilon}^{p q}}\right)-\frac{1}{\beta N} \sum_{k} \ln \left(1+\mathrm{e}^{-\beta\left(\tilde{\varepsilon}^{p q}+t_{k} \tilde{B}^{p q}\right)}\right) .
\end{aligned}
$$

One can test this result by introducing additional fields $h_{p q} \hat{B}^{p q}$ into Hamil-

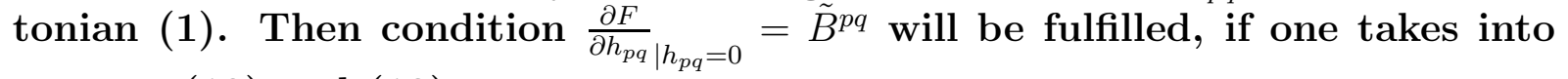
account (12) and (13).

In the paramagnetic phase $\tilde{B}^{41}=\tilde{B}^{31} \equiv \tilde{B}, \tilde{Q}^{41}=\tilde{Q}^{31} \equiv \tilde{Q}$. Hence, the full set of simultaneous equations defining the mean field approximation for thermodynamic quantities of Hubbard model consists of the equations (8) and (12) as well as of the condition for mean number of electrons per site

$$
\left\langle X^{33}+X^{44}\right\rangle=n
$$

One can show that standard condition for Hubbard operators

$$
\left\langle X^{11}+X^{33}+X^{44}\right\rangle=\left\langle X^{44}\right\rangle+\tilde{B}=1
$$

is fulfilled automatically.

Considered this result within the wide class of approximation for Hubbard model we noted that in the limit $U \rightarrow \infty$ it is equivalent to results obtained within the moment technique [23, 24] (see also [25]) which describes the shift of Hubbard bands on value depended on spin $\sigma$

$$
B_{\sigma}=\frac{1}{n_{\sigma}\left(1-n_{\sigma}\right)} \sum_{i \neq j} t_{i j}\left\langle a_{i \sigma}^{+} a_{j \sigma}\left(2 n_{i-\sigma}-1\right)\right\rangle .
$$

with $n_{\sigma}=\left\langle\hat{n}_{\sigma}\right\rangle$. One can show that there is clear correspondence between $B_{\sigma}$ and $\tilde{Q}^{p q}$ :

$$
\tilde{Q}^{41}=-n_{\uparrow} B_{\uparrow}, \quad \tilde{Q}^{31}=-n_{\downarrow} B_{\downarrow}
$$


But in MFA approach we have also equations for end parts for Green functions $\tilde{B}^{p q}\left(n_{\sigma}\right)$ which is needed for consideration of processes where ordered phase takes place as well as the equation for free energy (14) required for investigation of phase transitions of first order.

\section{Two-sublattice Hubbard model with local anhar- monicity}

The Hamiltonian of the two-sublattice Hubbard model with local anharmonicity which includes also an interaction of electrons with local anharmonic vibrations, described within the pseudospin formalism, reads (21])

$$
\begin{aligned}
& H=H_{e}+H_{s}+H_{e-s}+H_{s-s} \\
& H_{e}=-\mu \sum_{i, s}\left(\hat{n}_{i 1}^{s}+\hat{n}_{i 2}^{s}\right)+U \sum_{i}\left(\hat{n}_{i 1}^{\uparrow} \hat{n}_{i 1}^{\downarrow}+\hat{n}_{i 2}^{\uparrow} \hat{n}_{i 2}^{\downarrow}\right)+\sum_{i j} \sum_{s \alpha} t_{i j} a_{i s \alpha}^{+} a_{j s \alpha} \\
& H_{s}=-h \sum_{i}\left(S_{i 1}^{z}-S_{i 2}^{z}\right) \\
& H_{e-s}=g \sum_{i s}\left(\hat{n}_{i 1}^{s} S_{i 1}^{z}-\hat{n}_{i 2}^{s} S_{i 2}^{z}\right) \\
& H_{s-s}=-\frac{1}{2} \sum_{i, j} \sum_{\alpha \beta} J_{i j}^{\alpha \beta} S_{i \alpha}^{z} S_{j \beta}^{z}
\end{aligned}
$$

Here $H_{e}$ is the Hubbard Hamiltonian for two sublattices (subplanes in the case of the $\mathrm{YBa}_{2} \mathrm{Cu}_{3} \mathrm{O}_{7-\delta}$ structure), $H_{s}+H_{s-s}$ is a pseudospin part of the Hamiltonian similar to the Mitsui model used in the ferroelectrics theory; $H_{e-s}$ describes the interaction between the electron and pseudospin subsystems. The operators $\hat{n}_{i \alpha}^{s}$ and $S_{i \alpha}^{z}$ act at the site $i$ of the plane $\alpha(\alpha=1,2)$. $h$ describes the asymmetry of a two-minima potentials.

Similarly to that performed for the standard Hubbard model (1), let us introduce a cluster basis of states $\left|R_{i}\right\rangle \equiv\left|n_{i 1}^{\uparrow}, n_{i 1}^{\downarrow}, n_{i 2}^{\uparrow}, n_{i 2}^{\downarrow}\right\rangle \otimes\left|S_{i 1}^{z}, S_{i 2}^{z}\right\rangle$, or $\left|R_{i}\right\rangle \equiv\left|n_{i 1}^{\uparrow}, n_{i 1}^{\downarrow}, n_{i 2}^{\uparrow}, n_{i 2}^{\downarrow}, S_{i 1}^{z}, S_{i 2}^{z}\right\rangle$ (here $n_{i \alpha}^{\uparrow}, n_{i \alpha}^{\downarrow}$ denote eigenvalues of the operators $\hat{n}_{i \alpha}^{\uparrow}$ and $\hat{n}_{i \alpha}^{\downarrow}$ ), consisting of 64 vectors of state

$$
\begin{aligned}
& |1\rangle=|0,0,0,0, \uparrow, \uparrow\rangle \\
& \cdots \\
& |64\rangle=|1,1,1,1, \downarrow, \downarrow\rangle .
\end{aligned}
$$

Analysis of the thermodynamics of the model (18) without taking into account the electron transfer has been performed in [21], where the regions of ferroelectric pseudospin ordering were found and phase diagrams where built. Now we will investigate the influence of electron transfer on thermodynamic properties and phase transitions. Let us consider a pseudospin part of the Hamiltonian within the mean field approximation and an electron subsystem via the presented in the previous Section selfconsistent GRPA scheme.

The cluster basis of states consists of a direct product of two single-sublattice $|i, \alpha, R\rangle$ $\equiv\left|\hat{n}_{i \alpha}^{\uparrow}, \hat{n}_{i \alpha}^{\downarrow}, \hat{S}_{i \alpha}^{z}\right\rangle(\alpha=1,2)$ ones. Each single-sublattice set of states includes eight 
components at a given site $i$ and sublattice $\alpha$ :

$$
\begin{array}{lll}
|1\rangle=|0,0, \uparrow\rangle & |\tilde{1}\rangle=|0,0, \downarrow\rangle \\
|2\rangle=|1,1, \uparrow\rangle & |\tilde{2}\rangle=|1,1, \downarrow\rangle \\
|3\rangle=|0,1, \uparrow\rangle & |\tilde{3}\rangle=|0,1, \downarrow\rangle \\
|4\rangle=|1,0, \uparrow\rangle & |\tilde{4}\rangle=|1,0, \downarrow\rangle
\end{array}
$$

Let us introduce the Hubbard operators $X_{i \alpha}^{R S}=|i, \alpha, R\rangle\langle i, \alpha, S|$ in this basis. Then the operators $a_{i \uparrow \alpha}, a_{i \downarrow \alpha}, S_{i \alpha}^{z}$ are related to the X-operators as

$$
\begin{aligned}
& a_{i \uparrow \alpha}=X_{i \alpha}^{14}+X_{i \alpha}^{32}+X_{i \alpha}^{\tilde{1} \tilde{4}}+X_{i \alpha}^{\tilde{3} \tilde{2}}, \quad a_{i \downarrow \alpha}=X_{i \alpha}^{13}-X_{i \alpha}^{42}+X_{i \alpha}^{\tilde{1} \tilde{3}}-X_{i \alpha}^{\tilde{4} \tilde{2}} \\
& S_{i \alpha}^{z}=\frac{1}{2} \sum_{k=1}^{4}\left(X_{i \alpha}^{R R}-X_{i \alpha}^{\tilde{R} \tilde{R}}\right)
\end{aligned}
$$

Then Hamiltonian (18) can be easily rewritten as

$$
H=H_{0}+H_{\text {int }}+H_{s-s},
$$

where $H_{0}=\sum_{p=1}^{\tilde{4}} \sum_{i, \alpha} \varepsilon_{\alpha}^{p} X_{i \alpha}^{p p}$ with

$$
\begin{array}{ll}
\varepsilon_{1}^{1, \tilde{1}}=\mp \frac{h}{2}, & \varepsilon_{2}^{1, \tilde{1}}= \pm \frac{h}{2} \\
\varepsilon_{1}^{2, \tilde{2}}=-2 \mu+U \mp \frac{h}{2} \pm g, & \varepsilon_{2}^{2, \tilde{2}}=-2 \mu+U \pm \frac{h}{2} \mp g \\
\varepsilon_{1}^{3, \tilde{3}}=\varepsilon_{1}^{4, \tilde{4}}=-\mu \mp \frac{h}{2} \pm \frac{g}{2}, & \varepsilon_{2}^{3, \tilde{3}}=\varepsilon_{2}^{4, \tilde{4}}=-\mu \pm \frac{h}{2} \mp \frac{g}{2}
\end{array}
$$

and

$$
\begin{aligned}
& H_{i n t}=\sum_{i j, \alpha} t_{i j}\left(X_{i \alpha}^{41}+X_{i \alpha}^{23}+X_{i \alpha}^{\tilde{4} \tilde{1}}+X_{i \alpha}^{\tilde{2} \tilde{3}}\right) \times\left(X_{j \alpha}^{14}+X_{j \alpha}^{32}+X_{j \alpha}^{\tilde{1} \tilde{4}}+X_{j \alpha}^{\tilde{2} \tilde{3}}\right)+ \\
& +\sum_{i j \alpha} t_{i j}\left(X_{i \alpha}^{31}-X_{i \alpha}^{24}+X_{i \alpha}^{\tilde{3} \tilde{1}}-X_{i \alpha}^{\tilde{2} \tilde{4}}\right) \times\left(X_{j \alpha}^{13}-X_{j \alpha}^{42}+X_{j \alpha}^{\tilde{1} \tilde{3}}-X_{j \alpha}^{\tilde{4} \tilde{2}}\right)
\end{aligned}
$$

$H_{s-s}$ is left unchanged.

It should be noted that the model (18) does not take into account the transfer of electrons between the planes. Therefore, if the interplane interaction between pseudospins is absent $\left(J_{i i^{\prime}}^{12}=J_{i i^{\prime}}^{21}=0\right)$, and also owing to the system symmetry, the problem is reduced to two identical single-sublattice subproblems.

Due to the relation

$$
\left[S_{i \alpha}^{z}, X_{j \beta}^{p q}\right]=0
$$

and also when a splitting of the mean field type $S_{i \alpha}^{z} S_{j \beta}^{z}=\left\langle S_{i \alpha}^{z}\right\rangle S_{j \beta}^{z}+\left\langle S_{j \beta}^{z}\right\rangle S_{i \alpha}^{z}-\frac{1}{2}\left\langle S_{i \alpha}^{z}\right\rangle\left\langle S_{j \beta}^{z}\right\rangle$ is used, the effective Hamiltonian of the problem reads

$$
H_{e f f}=H_{0}+\sum_{\{p q\}} \sum_{i \alpha} \tilde{Q}_{\alpha}^{p q} \hat{B}_{i \alpha}^{p q}-\sum_{i \alpha \alpha^{\prime}} J_{\alpha \alpha^{\prime}}\left\langle S_{\alpha^{\prime}}^{z}\right\rangle S_{i \alpha}^{z},
$$

where $J_{\alpha \alpha^{\prime}}=\sum_{i} J_{i i^{\prime}}^{\alpha \alpha^{\prime}}$.

When writing equations of self-consistent GRPA approach for the two-sublattice model (18) in the approximation of independent subbands [8] and at $U=\infty$, we can use expressions of the previous Section. All the quantities $\widetilde{Q}_{\alpha}^{p q}, \tilde{B}_{\alpha}^{p q}$, etc. get an auxiliary index 
indicating the subplane $\alpha=1,2$. For instance, owing to the presence of pseudospin degrees of freedom, in addition to $B_{\alpha}^{p q}$ we also have $B_{\alpha}^{\tilde{p} \tilde{q}}$. Then the summation $\sum_{\{p q\}}$ means the summation over $p q=41, \tilde{4} \tilde{1}, 31, \tilde{3} \tilde{1}$. An additional contribution to the free energy $\tilde{D}^{p q}$ we should change by $\tilde{D}_{\alpha}^{p q}$, where

$$
\tilde{D}_{\alpha}^{p q}=\frac{1}{\beta N} \sum_{k} \ln \left(1+\mathrm{e}^{-\beta \tilde{\varepsilon}_{\alpha}^{p q}}\right)-\frac{1}{\beta N} \sum_{k} \ln \left(1+\mathrm{e}^{-\beta\left(\tilde{\varepsilon}_{\alpha}^{p q}+t_{k} \tilde{B}_{\alpha}^{p q}\right)}\right)
$$

with

$$
\tilde{\varepsilon}_{\alpha}^{p q}=\varepsilon_{\alpha}^{p}-\varepsilon_{\alpha}^{q}-\tilde{Q}_{\alpha}^{\overline{p q}}
$$

The expression for a free energy now reads

$$
F=\mu n-T \ln S p\left(\mathrm{e}^{-\beta\left(H_{e f f}\right)}\right)-\sum_{\substack{\{p q\} \\ \alpha}} \tilde{Q}_{\alpha}^{p q} \tilde{B}_{\alpha}^{p q}+\sum_{\substack{\{p q\} \\ \alpha}} \tilde{D}_{\alpha}^{p q}+\frac{1}{2} \sum_{\alpha \alpha^{\prime}} J_{\alpha \alpha^{\prime}}\left\langle S_{\alpha}^{z}\right\rangle\left\langle S_{\alpha^{\prime}}^{z}\right\rangle
$$

The system of equations to be solved

$$
\begin{aligned}
& \tilde{B}_{\alpha}^{p q}=\frac{S p\left(\hat{B}_{\alpha}^{p q} \mathrm{e}^{\left.-\beta H_{e f f}\right)}\right.}{S p\left(\mathrm{e}^{\left.-\beta H_{e f f}\right)}\right.}-\tilde{L}^{\overline{p q}} \\
& \tilde{Q}_{\alpha}^{p q}=\frac{1}{N} \sum_{k} t_{k} n_{+}\left(\tilde{\varepsilon}_{\alpha}^{p q}+t_{k} \tilde{B}_{\alpha}^{p q}\right)
\end{aligned}
$$

should be supplemented by a standard identity for Hubbard operators $\left\langle\sum_{p=1}^{\tilde{4}} X_{\alpha}^{p p}\right\rangle=1$, by the equation for the pseudospin mean value $\left\langle S_{\alpha}^{z}\right\rangle=S p\left(\hat{S}_{\alpha}^{z} \mathrm{e}^{-\beta H_{\text {eff }}}\right) / S p\left(\mathrm{e}^{-\beta H_{\text {eff }}}\right)$, the equation for the chemical potential $\sum_{\alpha=1}^{2}\left\langle X_{\alpha}^{33}+X_{\alpha}^{44}+X_{\alpha}^{\tilde{3} \tilde{3}}+X_{\alpha}^{\tilde{4} \tilde{4}}\right\rangle_{H_{e f f}}=n$ as well as by the equalities $\left\langle X_{\alpha}^{33}\right\rangle=\left\langle X_{\alpha}^{44}\right\rangle,\left\langle X_{\alpha}^{\tilde{3} \tilde{3}}\right\rangle=\left\langle X_{\alpha}^{\tilde{4} \tilde{4}}\right\rangle$ in paramagnetic case.

\section{Numerical calculations and results}

In paper [22], where the pseudospin-electron model with zero transfer was studied, the existence of the phase separation resulting from the direct interaction between pseudospins was revealed. This effect takes place, as it was shown, in the certain region of the model parameters values in the regime of the fixed electron concentration $(n=$ const $)$. On the other hand, the simple pseudospin-electron model or the more complicated model (18) introduced for a description of possible phase instabilities in HTSCs of the YBaCuO type do not take into account explicitly the existence of $\mathrm{CuO}$ chains which are a reservoir supplying a charge carriers into the superconducting planes.

Therefore, the model can be considered in two regimes: a) constant concentration, and b) constant chemical potential (the fixed value of the chemical potential $\mu$ is sustained by structural elements which are not taken into account within the model).

The ground state diagram in absence of direct pseudospin-pseudospin interaction and transfer $\left(J_{\alpha \beta}=0, t_{i j}=0\right)$ is shown in Fig.11. At $T=0$ the chemical potential coincide with the line ABCD at any value of concentration $n$. When temperature is different from zero, the chemical potential lies to the right of $\mathrm{ABCD}$ at $n \sim 0$ and to the left of it at 


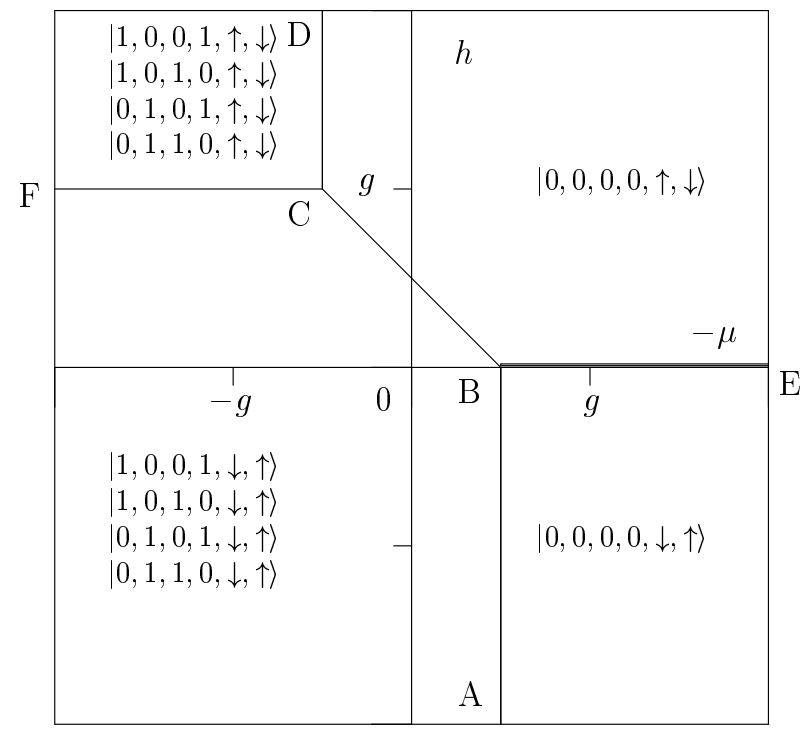

Figure 1: Ground state diagram in the $\mu$ - $h$ plane in absence of direct pseudospin $\left(J_{\alpha \beta}=0\right)$ interaction $\left(J_{\alpha \beta}=0\right)$ and electron transfer $\left(t_{i j}=0\right) . U=\infty$. The electron and pseudospin configurations are shown.

$n \sim 2$. In the $\mu=$ const regime, the chemical potential is given by the line parallel to the ordinate axis.

Analysis performed in the spirit of the mean field theory [21] has shown that in the regime of a fixed value of the chemical potential, the region of ferroelectric type ordering of pseudospins lies in the vicinity of the FCBE curve. This curve is a boundary between the ground states with the pseudospin configurations $|\uparrow \downarrow\rangle$ and $|\downarrow \uparrow\rangle$. If the interplane interaction $J_{12}$ is different from zero, the mean value $\eta=\left\langle S_{1}^{z}+S_{2}^{z}\right\rangle$ is the order parameter: $\eta=0$ in the paraphase and $\eta \neq 0$ in the ferroelectric phase. At $J_{12}=0$ we deal with two independent single-sublattice problems, and the order parameter is zero; however, a jump of the parameter $\xi=\left\langle S_{1}^{z}-S_{2}^{z}\right\rangle$ takes place.

Figs.2.3 illustrate the influence of electron subsystem on a appearance of the phase diagrams. Let us note that in the Mitsui model (to which one can pass by putting $g=0$ and $\left.t_{i j}=0\right)$ the phase diagram has a form of a curve symmetric with respect to $h$. At "switching on" the interaction between the electron and pseudospin subsystems $g$, the center of a region with ferroelectric ordering shifts from 0 to $g$ in accordance with the broken line FCBE position when the chemical potential is changed (Fig.1). Besides, the phase diagram becomes asymmetric with respect to the center of the pseudospin ordering region. As it has been already noted, at $J_{12}=0$ the ferroelectric phase does not exist (figs.2]b,3 b, 3]c), a discontinuous change of $\xi$ takes place.

Let us now consider the influence of electron transfer on the phase transitions in the simplest approximation Hubbard-I. It has been shown in [7] that at $U \rightarrow \infty$ in a one-sublattice case for the Hubbard model with local anharmonicity, one has in HubbardI approximation two subbands, separated by the gap equal to $g$ : $\varepsilon^{31}(\mathbf{k})=\varepsilon^{41}(\mathbf{k})=$ $\frac{g}{2}+t_{\mathbf{k}}\left\langle X^{44}+X^{11}\right\rangle_{0}$ and $\varepsilon^{\tilde{3} \tilde{1}}(\mathbf{k})=\varepsilon^{\tilde{4} \tilde{1}}(\mathbf{k})=-\frac{g}{2}+t_{\mathbf{k}}\left\langle X^{\tilde{4} \tilde{4}}+X^{\tilde{1} \tilde{1}}\right\rangle_{0}$. In the considered in this paper approximation, these subbands are shifted by $\tilde{Q}^{p q}$; the mean values of the Hubbard 

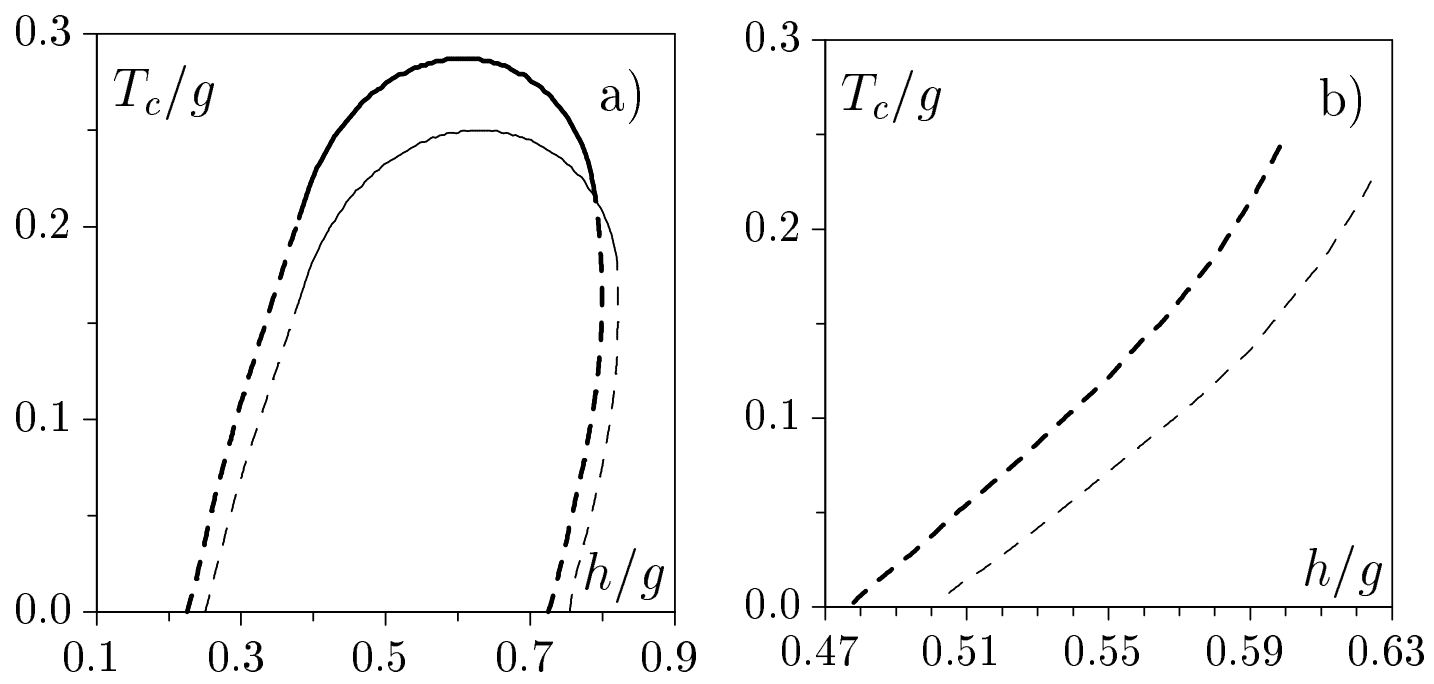

Figure 2: Dependence of temperature of the phase transition $T_{c}$ on the parameter $h$ at different values of interaction parameters $J_{11}$ and $J_{12}$ in the regime $\mu=$ const $=0$. Thick lines correspond to the case $t_{i j} / g=0.2$; thin lines correspond to the case $t_{i j}=0$. a) $\left.J_{11}=J_{12}=g / 2, \mathrm{~b}\right) J_{11}=g, J_{12}=0$. Phase transitions are of the second order (solid lines) or of the first order (dashed lines).
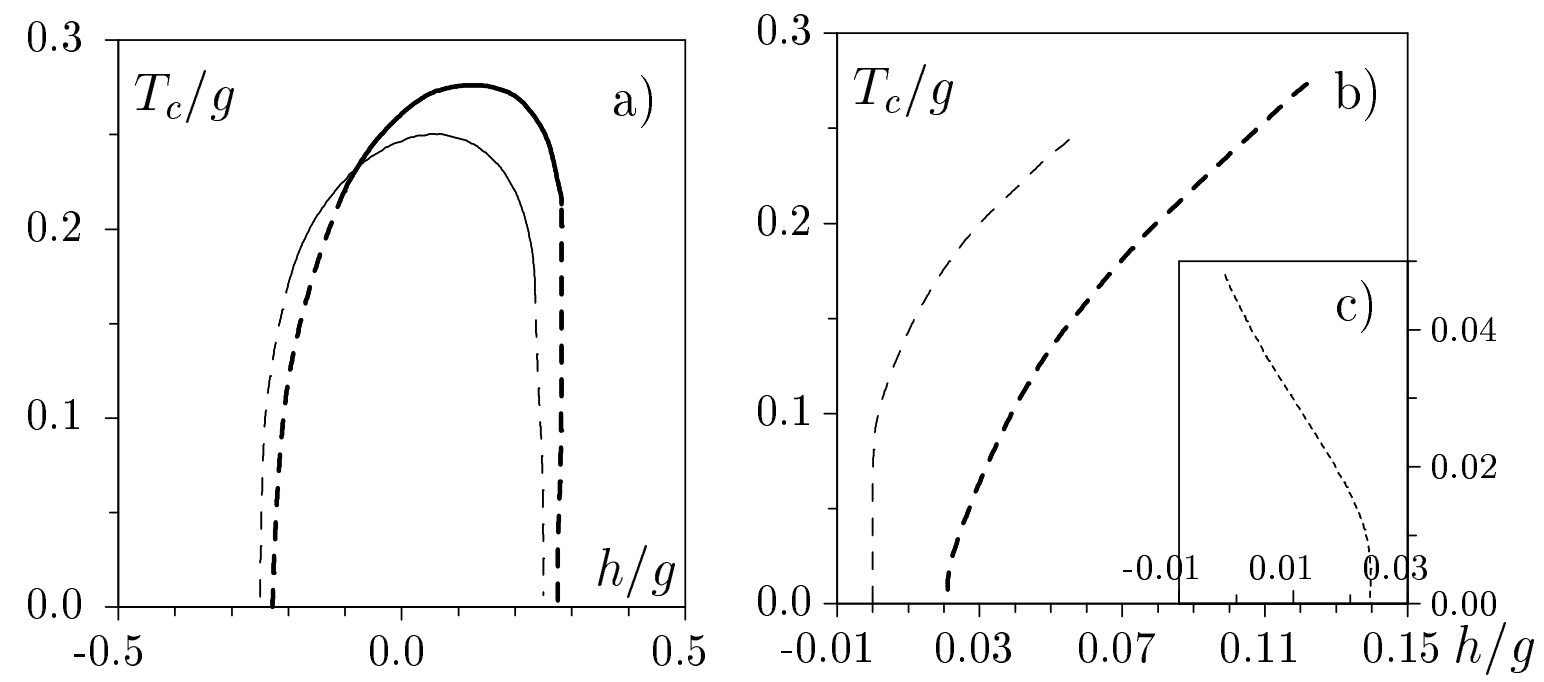

Figure 3: Dependence of temperature of the phase transition $T_{c}$ on the parameter $h$ at different values of interaction parameters $J_{11}$ and $J_{12}$ in the regime $\mu=$ const $=-g$. Thick lines correspond to the case $t_{i j} / g=0.2$; thin lines correspond to the case $t_{i j}=0$. a) $J_{11}=J_{12}=g / 2$, b) $J_{11}=g, J_{12}=0$. c) $J_{11}=J_{12}=0, t_{i j} / g=0.2$. Phase transitions are of the second order (solid lines) or of the first order (dashed lines). 

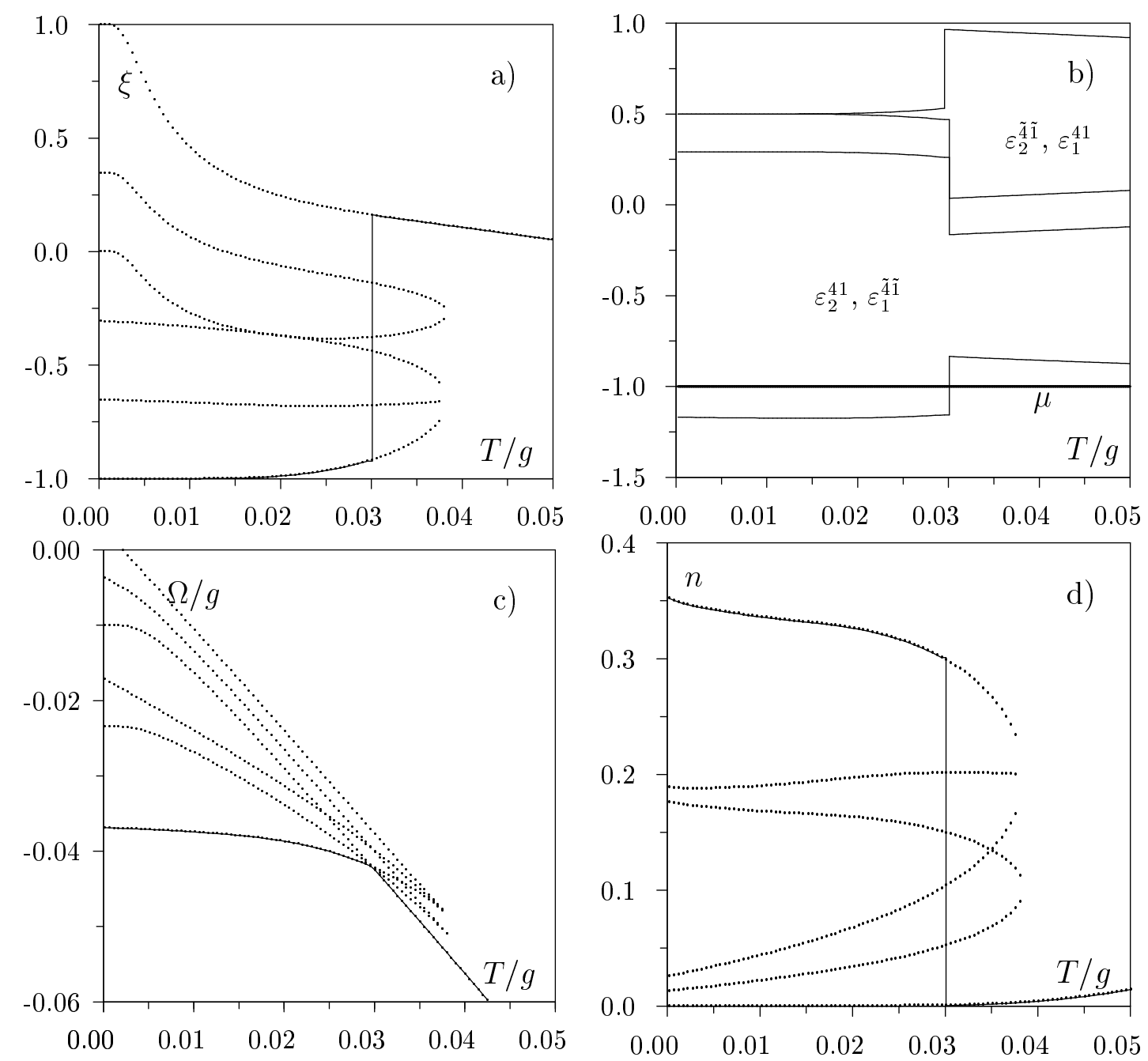

Figure 4: Dependence of the order parameter $\xi(\mathrm{a})$, band edges (b), thermodynamic potential (c), and electron concentration (d) on temperature in the regime $\mu=$ const $=$ $-g$. The parameters values are: $J_{11}=J_{12}=0, T / g=0.1, t_{i j} / g=0.2, h / g=0.01$.

operators are not calculated in the zero-order approximation but found self-consistently. In the case shown on Fig.2 $(\mu=0)$, the chemical potential lies right in between of the mentioned bands. The number of charge carriers slightly changes with temperature and parameter $h$. The transfer can facilitate, but by itself is not able to cause the phase transition.

At $\mu=-g$ (Fig.[3) the chemical potential lies near the edge of the lower band. Even unsignificant change in the spectrum can make the chemical potential leave the band (Fig. $1 \mathrm{~b}$ ) and provokes jump of parameter $\xi$ (Fig.4a). In this case, interaction via electron transfer alone can cause the phase transition in the system. Fig. 四c indicates that the phase transition of the first order takes place. The behaviour of electron concentration here is quite specific (Fig. $4 \mathrm{~d}$ ), when it jumps from a certain value at $T=0$ up to 0 when the chemical potential leaves the band. On the other hand, the existence of instable and metastable branches in Fig. 1 indicates that the chemical potential is complicated function 

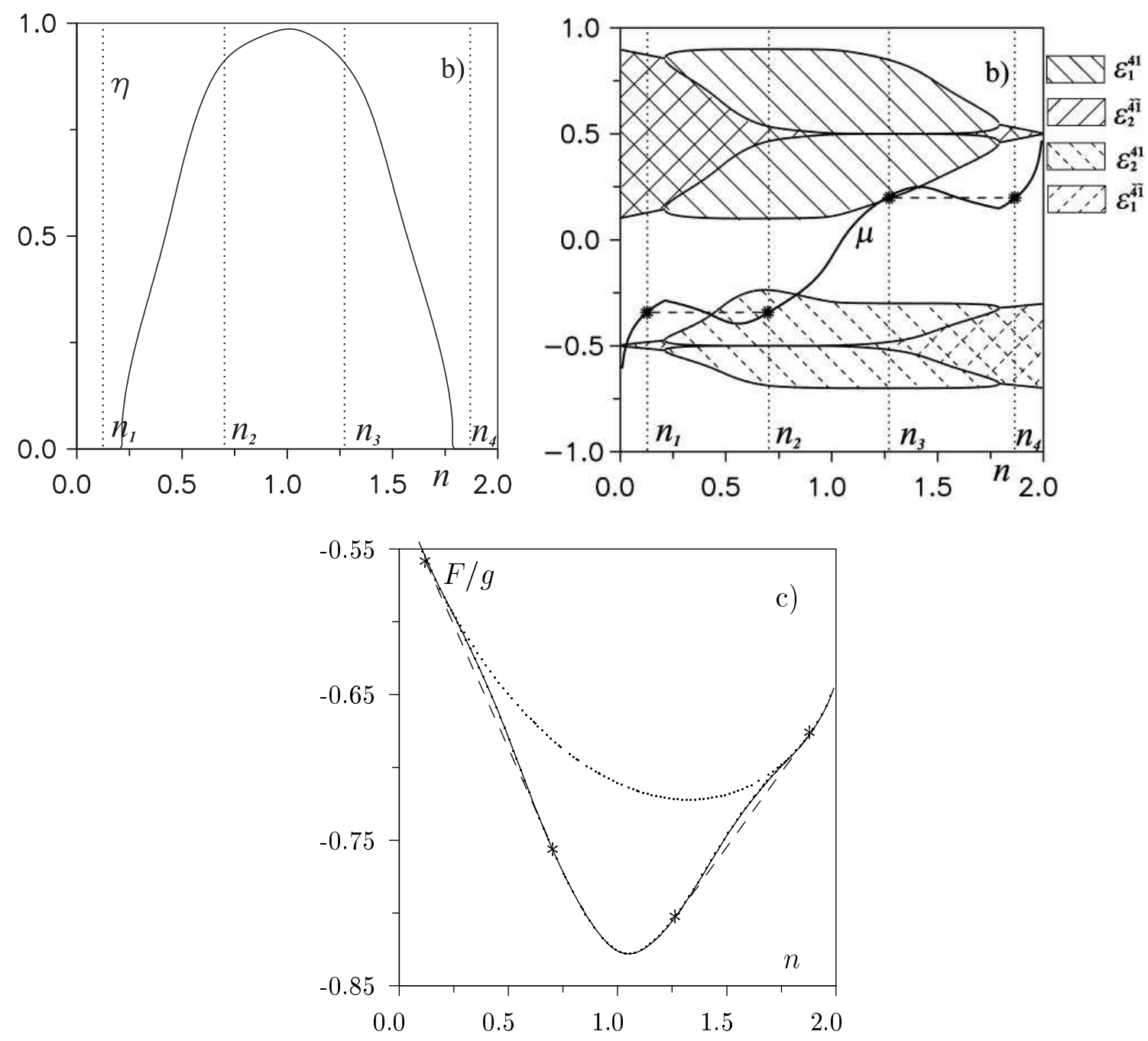

Figure 5: Dependence of the order parameter $\eta(\mathrm{a})$, band spectrum (b), and free energy (c) on concentration. The parameters values are: $J_{11}=J_{12}=g / 2, T / g=0.1, t_{i j} / g=0.1$, $h / g=0.5$.

of concentration. Therefore the situation when at a certain value of $n$ the function $\mu(n)$ has a jump becomes possible. In this case we have separation to two phases with different concentration, similarly to a seen in the $P(V)$ diagram separation of vapour into gas and liquid in the gas-liquid phase transition.

Fig.5 shows that even though the solution of the system of equations indicates that the ferroelectric phase arose (Fig.5a), the separation takes place before that (Fig.5b). It is also indicated by a certain concavity in the free energy behaviour (tangent dashed lines in Fig.5c link the points with concentration values $n_{1}, n_{2}$ and $n_{3}, n_{4}$ on which the separation take place). Hence, in the $n=$ const regime we can talk only about the separation on the paraelectric and ferroelectric phases at concentration values $n_{1}<n<n_{2}$ and $n_{3}<n<n_{4}$. The ferroelectric phase exists (in the case considered in Fig.5) in the concentration range $n_{2}<n<n_{3}$. Fig.6a and $6 \mathrm{~b}$ show the boundaries of the separated phase with and without 

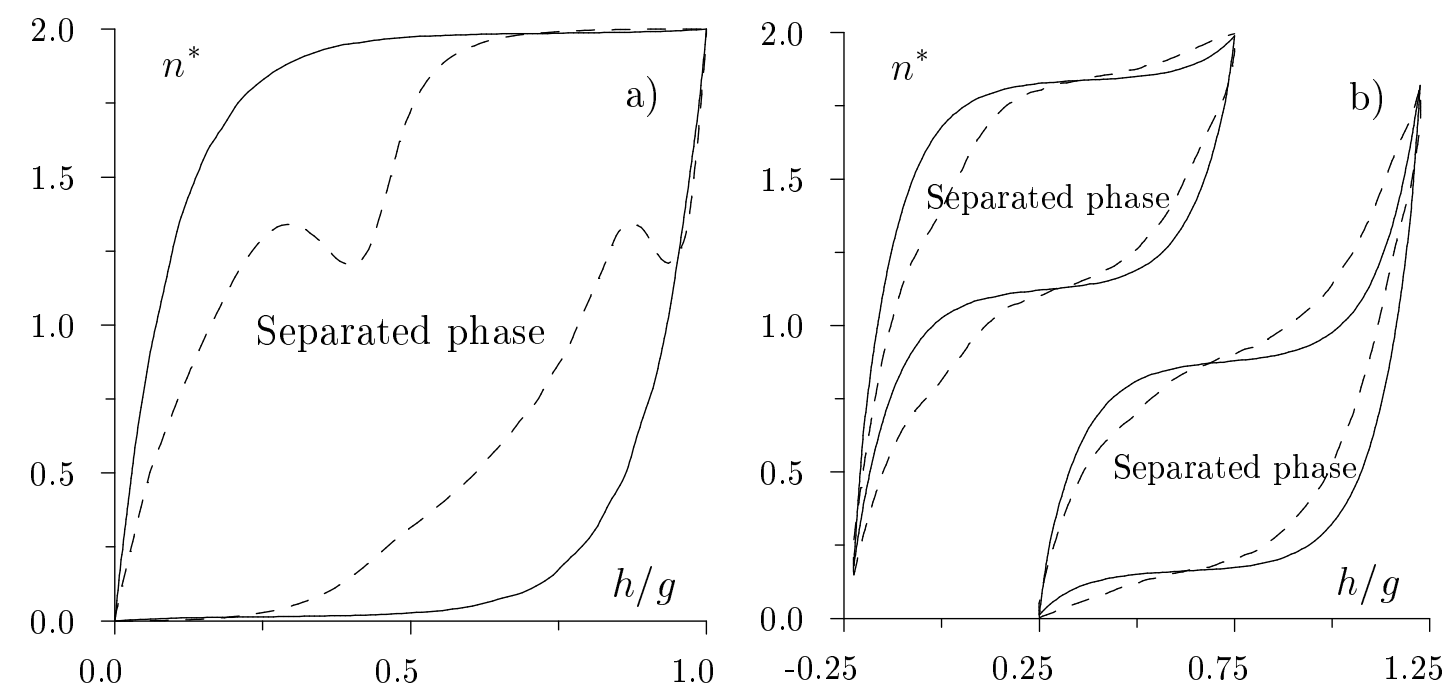

Figure 6: Regions of phase separation at different values of interaction $J_{\alpha \beta}, T / g=0.1$. Thick lines correspond to the case $t_{i j}=0$; dashed lines correspond the the case $t_{i j} \neq 0$. a) $J_{11}=g, J_{12}=0, t_{i j} / g=0.2$; b) $J_{11}=J_{12}=g / 2, t_{i j} / g=0.1$.

transfer, positions of which are temperature dependent. At $T=0$ the region of phase separations reaches the sides of the $0 \leq h / g \leq 1,0 \leq n^{*} \leq 2$ [22] rectangle. The transfer influence is ambiguous. In the first case (6a) it narrows the region of separations, whereas in the second case (6 $\mathrm{b}$ ), the transfer only changes the shape of the region boundaries. It should be noted that in the presented figures, the high temperature is taken; therefore, at chosen values of model parameters and in the absence of direct pseudospin-pseudospin interaction, the separation does not exist.

\section{Conclusions}

The mean field approximation, being the simplest one in the theory of many-particle systems, is not trivial for the models with strong correlations of charge carriers. In this case, the effective field is created by transfer of electrons from site to site, not by regular averaging of a direct interaction. In contrast to the case of Fermi-systems, we have to solve here not only the equation for the self-energy part, but also some extra equations for the averages of the Hubbard operators [1]. In this paper we show that this approach means a selection of diagrams with only one sum over the wavevector $\mathbf{k}$ and frequency $\omega$ in expressions for the self-energy part and averages and is a generalization of the GRPA. Obtained shift of lower Hubbard band corresponds to results of the moment technique [23, 24, 25] in paraphase and in the limit $U \rightarrow \infty$. The MFA equations for self-consistency parameters and expressions for thermodynamic functions allow one to analyze the equilibrium states and phase diagrams for the systems with strong correlation.

In 10 it has been shown how to obtain an equation of the dynamic mean field approximation $(d=\infty)$ for the Falicov-Kimball model by means of summation of diagrams and selecting the single-site contributions. Nevertheless, the question of analytical con- 
sideration of such contributions remains in general still open. Hence, we hope that a self-consistent approach, similar to the presented one, will give us a clue how to solve a single-site problem in the infinite dimensions for the another examples of models with short-range electron interaction.

The mean field approximation applied to the two-sublattice model with local anharmonicity indicates a possibility of the phase separation in the system. It has been shown in [22] that the direct pseudospin-pseudospin interaction is sufficient to make a system divide into regions with different concentration of charge carriers. Instabilities with respect to phase separation were found in the ferromagnetic Condo model (see for example 26]), single-sublattice pseudospin-electron model with $U=0$ and without direct interaction between pseudospins [27]. From this point of view, our paper shows that the electron transfer not only does not destroy the separation regions, but facilitates process separation and even is able to form separated phases by itself. This effect takes place when the chemical potential $\mu$ lies near the band edge, and the change of energy spectrum (due to ordering of pseudospins) in the $\mu=$ const regime moves the chemical potential into or out of the band. One can conclude that the electron (hole) transfer in the pseudospin-electron model creates an effective interaction between pseudospins. It is qualitatively different from the standard one $J_{i j} S_{i}^{z} S_{j}^{z}$ [9], but its role is similar.

The problem of applicability of the approach proposed in this paper, as well as of the GRPA itself, requires a thorough analysis. The approach can be improved at the expense of complication of loop diagrams and vertices. Here the magnon Green function

$g^{43}(\mathbf{k}, \omega)$ arise. Taking into account the magnon contributions is especially important at $U \neq \infty$, since without them the system of equations for the averages of the Hubbard operators becomes dependent on the priority rules. These problems are the subject of our subsequent studies.

\section{References}

[1] Izyumov Yu.A., Letfulov B.M. and Shipitsyn E.V., A mean-field-type approximation for the $(t-J)$ model. // J. Phys.: Condens. Matter, 1994, vol. 6, p. 5137-5154.

[2] Hubbard J., Proc. R. Soc. London, 1963, Ser A vol. 276, p. 238.

[3] Abrikosov A.A., Gorkov L.P. and Dzyaloshinskii I.E., Methods of quantum field theory in statistical physics. // 1975, New York: Dover.

[4] Izyumov Yu.A., Letfulov B.M., Shipitsyn E.V. and Chao K.A., A theory of ferromagnetism in the Hubbard model with infinite Coulomb interaction. // Int. Journ. Mod. Phys. B, 1992, vol. 6, No. 21, p. 3479-3514.

[5] Izyumov Yu.A., Letfulov B.M., and Shipitsyn E.V., Bartkowiak M. and Chao K.A., Theory of strongly correlated electron systems on the basis of diagrammatic technique for Hubbard operators. // Phys. Rev. B, 1992, vol. 46, No. 24, p. 15697-15711.

[6] Izyumov Yu.A., Letfulov B.M. and Shipitsyn E.V., Generalized random-phase approximation in the theory of strongly correlated systems. // J. Phys.: Condens. Matter, 1992, vol. 4, p. 9955-9970. 
[7] Stasyuk I.V., Shvaika A.M. Dielectric properties and electron spectrum of the Müller model in the HTSC theory. // Acta Physica Polonica A, 1993, vol. 84, No 2, p. 293313.

[8] Stasyuk I.V., Shvaika A.M. Dielectric instability and local anharmonic model in the theory of high- $\mathrm{T}_{c}$ superconductivity. // Physica C, 1994, vol. 235-240, p. 2173-2174.

[9] Stasyuk I.V., Shvaika A.M. and Danyliv O.D. Dielectric instability and charge ordering in the local anharmonic model of high $T_{c}$ superconductors. // Molecular Physics Reports, 1995, vol. 9, p. 61-75.

[10] Letfulov B.M., Strongly correlated Falicov-Kimball model in infinite dimensions // Eur. Phys. J. B, 1998, vol. 4, p. 447-457.

[11] Letfulov B.M., A theory of double exchange in infinite dimensions. // Eur. Phys. J. B, 1998, vol. 4, p. 195-203.

[12] Stasyuk I.V., Shvaika A.M. Pseudospin-electron model in large dimensions. // J. Phys. Studies, 1999, vol. 3, No. 2, p. 177-183.

[13] Ruani G., Taliani C., Muccini M., Conder K., Kaldis E., Keller H., Zech D., Muller K.A. Apex anharmonicity observed by Raman scattering in ${ }^{18} \mathrm{O}$ substituted $\mathrm{YBa}_{2} \mathrm{Cu}_{3} \mathrm{O}_{6+x}$. // Physica C, 1994, vol. 226, p. 101-105.

[14] Mihailovic D. and Heeger A.J., Pyroelectric and piezoelectric effects in single crystals of $\mathrm{YBa}_{2} \mathrm{Cu}_{3} \mathrm{O}_{7-\delta}$. // Solid State Comm., 1990, vol. 75, p. 319-323.

[15] Grachev A.I. and Pleshkov I.V., Pyroelectric voltage in YBCO thin films.// Solid State Comm., 1997, vol. 101, No. 7, p. 507-512.

[16] Mustre de Leon J., Conradson S.D., Batistic' I., Bishop A.R., Raistrick I.D., Aronson M.C., and Garzon F.H. Axial oxygen-centered lattice instabilities in $\mathrm{YBa}_{2} \mathrm{Cu}_{3} \mathrm{O}_{7}$ : An application of the analysis of extended x-ray-absorbtion fine structure in anharmonic systems. // Phys.Rev.B, 1992, vol. 45, p.2447-2457.

[17] Conradson S.D., Raistrick I.D. The axial oxygen atom and superconductivity in $\mathrm{YBa}_{2} \mathrm{Cu}_{3} \mathrm{O}_{7}$. // Science, 1989, vol. 243, No 4896, p. 1340.

[18] Müller K.A. // Phase transitions, 1988 (Special issue).

[19] Stasyuk I.V., Slobodyan P.M. The diagrammatic technique for Hubbard operators. // Theor.Math.Phys. USSR, 1974, vol.19, p. 616 [TMF., 1974, vol. 19, No 3, p. 423-428 (in Russian)], Westwenski B. // Phys.Lett.A, 1973, vol.44, p. 27.

[20] Izyumov Yu.A., Skryabin Yu.N. Statistical Mechanics of Magnetically Ordered Systems // 1989, New York: Consultants Bureau.

[21] Danyliv O.D. Phase transitions in the two-sublattice pseudospin-electron model of high temperature superconducting systems. // Physica C, 1998, vol. 309, p. 303-314.

[22] Stasyuk I.V., Havrylyuk Yu. Phase separation in pseudospin-electron model with direct interaction between pseudospins. // Cond. Mat. Phys., 1999, vol. 2, No 3 (19). 
[23] Harris A.B. and Lange R.V. Single-particle excitations in narrow energy bands. // Phys.Rev., 1967, vol. 157, No 2, p.295-314.

[24] Eskes H., Oles' A.M., Meinders M.B.J. and Stephan W. Spectral properties of Hubbard bands. // Phys.Rev.B, 1994, vol. 50, No 24, p.17980-18002.

[25] Pothoff M., Herrmann T., Wegner T., and Nolting W. The momentum sum rule and its consequences for ferromagnetism. // Phys.Stat.Sol.(b), 1998, vol. 210, p.199-227.

[26] Moreo A., Avella A., and Cuoco M. Numerical studies of strongly correlated electron systems. In "Lectures on the physics of highly correlated electron systems". Ed. by Manchini F. // 1998, New York: Woodbury.

[27] Stasyuk I.V., Shvaika A.M., Tabunshchyk K.V. Thermodynamics of pseudospinelectron model without correlations. // Cond. Mat. Phys., 1999, vol. 2, No 1 (17), p. 109-132. 